\title{
COMPARISON LOAD MODELS IN HARMONIC FLOWS
}

\author{
V.F. Corasaniti, R. Bianchi, F. Viollaz
}

IITREE-LAT, Argentina

\begin{abstract}
With the increase of nonlinear loads in the distribution systems, it is important to carry out studies of harmonic flows with adequate models and to establish the possible resonances which can appear. This work has the aim to analize the influence of load model in the simulations, so that simulations with the load model CIGRE will be performed in contrast to the conventional model.
\end{abstract}

Keywords: Harmonics-Models-SimulationDistortion

\section{I.-INTRODUCTION}

For the last years an important increase of nonlinear loads in the distribution systems has been produced. It has caused the increase of waveform voltage and current supply distortions with the consequent loss in the power quality. Also damage of the components of the networks has been produced such as in user's equipment.

With the aim to study the distortion in the network studies of harmonic flows with apropiate models for that, these simulations will be carried out with the Electromagnetics Transients program ATP (Alternative Transients Program).

In this case, it will be made a study over a network of AT/MT (High voltage/ Medium voltage) $(132 / 13.2 \mathrm{kV})$, as, it is shown in Figure 1 , which has installed capacitor banks in different substations.

The purpose of this study is to get the impedance versus frequency in different system points with both load models, using the capacity of ATP to make frequency scan.

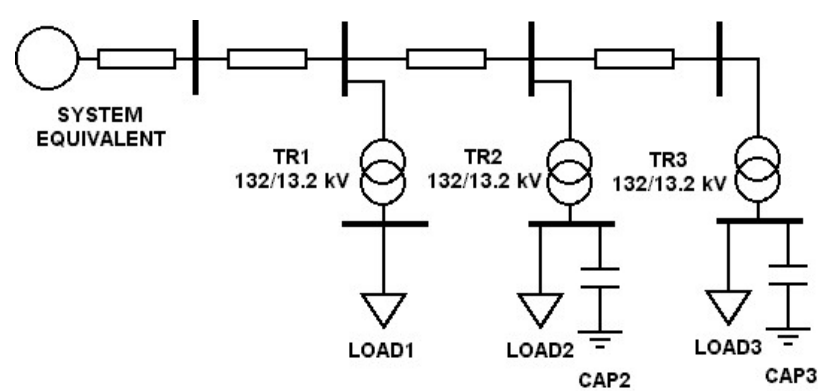

FIGURE1- System to analize
Then it will be studied the distortions which appear due to the presence of harmonic sources in the network, using other capacities of program, (HFS, Harmonic Frequency Scan), which allow to introduce harmonics through current sources of amplitude, frecuency and phase established by the user.

\section{II.-DEVELOPMENT}

Figure 2 shows the scheme of the network made with the ATPDRAW, a draw pre-proccesor for the ATP.

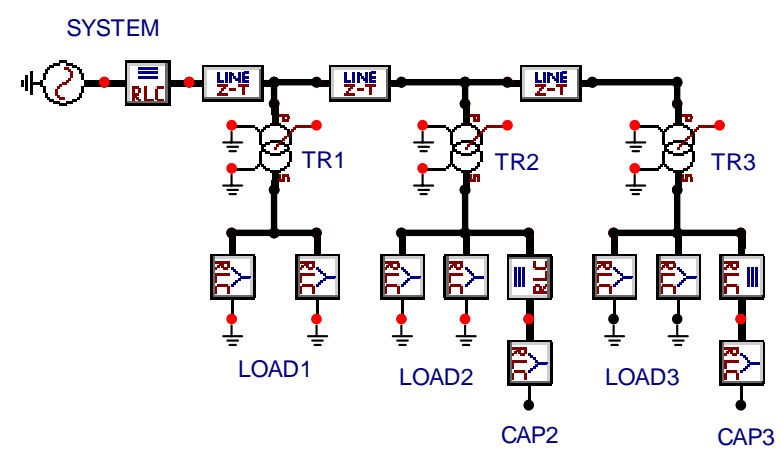

FIGURE2- Scheme of the network at ATP

The supply system of the network is represented with an equivalent at $132 \mathrm{kV}$ through short-circuit reactance at that point, connected there to three other substations under study through underground cables at $132 \mathrm{kV}$. The loads connected in bus of $13.2 \mathrm{kV}$ of each substation are from different values and each one has different degrees of reactive compensation.

Then it is carried out a description of adopted models and of data of each of the components used in the simulations.

\section{II.1) Underground cables:}

The characteristics of these cables are shown in Table1. Despite their short lengths, it is neccesary the representation through distribuited parameters line models because of the correction of the constants with the frecuency. Distribuited parameters and transposed line model (Clarke) which is at ATP were used. 
TABLE1- Parámeters of cables

\begin{tabular}{|c|c|c|c|}
\hline Cable & SYST-TR1 & TR1-TR2 & TR2-TR3 \\
\hline $\begin{array}{c}\text { Rated } \\
\text { Voltage }[\mathrm{kV}]\end{array}$ & 132 & 132 & 132 \\
\hline $\mathbf{r}[\Omega / \mathbf{k m}]$ & 0.052 & 0.059 & 0.063 \\
\hline $\mathbf{r}_{0}[\Omega / \mathbf{k m}]$ & 0.58 & 0.592 & 0.597 \\
\hline $\mathbf{L}[\mathrm{mH} / \mathbf{k m}]$ & 0.36 & 0.386 & 0.37 \\
\hline $\mathrm{L}_{0}[\mathrm{mH} / \mathrm{km}]$ & 0.573 & 0.608 & 0.627 \\
\hline $\mathbf{C}[\mu \mathrm{F} / \mathrm{km}]$ & 0.391 & 0.37 & 0.36 \\
\hline $\mathbf{C}_{0}[\mu \mathrm{F} / \mathrm{km}]$ & 0.391 & 0.37 & 0.36 \\
\hline Length. $[\mathrm{km}]$ & 2.4 & 1.82 & 2.75 \\
\hline
\end{tabular}

II.2) Transformers:

It was not taken into account the parameter variations of transformers with the frequency. It was used a conventional model which does not have variation of resistence of winding with frequency.

Table 2 shows their characteristics.

TABLE2- Parámeters of transformers

\begin{tabular}{|c|c|c|c|c|c|}
\hline TR & $\begin{array}{l}\text { Rated } \\
\text { Power } \\
\text { [MVA] }\end{array}$ & $\begin{array}{c}\text { Primary } \\
\text { Voltage } \\
{[\mathrm{kV}]}\end{array}$ & $\begin{array}{c}\text { Secondary } \\
\text { Voltage } \\
{[\mathrm{kV}]}\end{array}$ & Connection & $\begin{array}{c}\mathrm{X} \\
{[\%]}\end{array}$ \\
\hline TR1 & 20 & 132 & 13.2 & $Y_{\underline{I}} Y_{\frac{1}{I}}$ & 13.02 \\
\hline TR2 & 40 & 132 & 13.2 & $Y_{\underline{I}} Y_{\bar{I}}$ & 12.45 \\
\hline TR3 & 80 & 132 & 13.2 & $Y_{\frac{1}{2}} Y_{\frac{1}{I}}$ & 27 \\
\hline
\end{tabular}

II.3) Loads:

As it was said, the purpose of this work is to compare the results of simulations with two load models.

One of them does not have impedance variation of load with the frequency, (conventional model), while the other is a suggested model by CIGRE (1) for this kind of studies, which has variations of impedance load with the frequency.

Figure 3 shows the conventional single phase model.

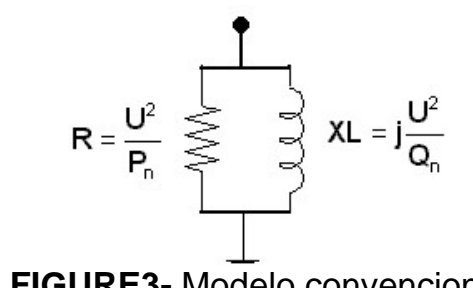

Figure 4 shows the single-phase model suggested by CIGRE.
The values of power demands in each subtation are shown in Table3.

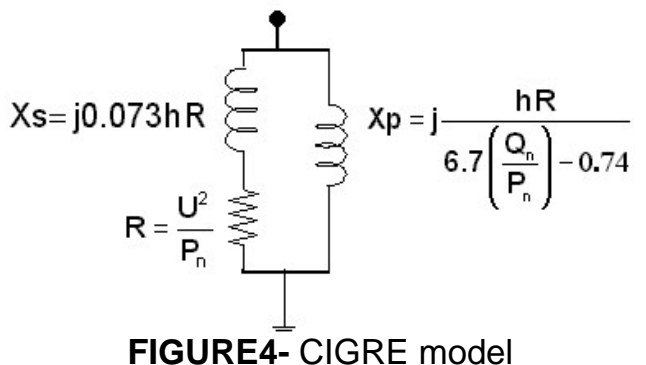

TABLE3- Power demands

\begin{tabular}{|c|c|c|c|}
\hline TR & $\begin{array}{c}\mathbf{P}_{\mathbf{n}} \\
{[\mathbf{M W}]}\end{array}$ & $\begin{array}{c}\mathbf{Q}_{\mathbf{n}} \\
{[\mathbf{M V A r}]}\end{array}$ & $\begin{array}{c}\mathbf{S}_{\mathbf{n}} \\
{[\mathbf{M V A}]}\end{array}$ \\
\hline TR1 & 13 & 6.5 & 14.5 \\
\hline TR2 & 13 & 6.5 & 14.5 \\
\hline TR3 & 26 & 10.86 & 28.2 \\
\hline
\end{tabular}

II.4) Reactors and capacitors:

These were modelled like concentrated parameters, without taking into account any variation of inductance or capacitance with frecuency.

The values of reactive capacitor banks installed for power factor compensation in each subtation are shown in Table4.

TABLE4- Capacitors data

\begin{tabular}{|c|c|c|c|c|}
\hline TR & $\begin{array}{c}\text { Rated } \\
\text { Voltage } \\
{[\mathbf{k V}]}\end{array}$ & $\begin{array}{c}\text { Rated } \\
\text { Current } \\
{[\mathbf{A}]}\end{array}$ & $\mathbf{N}^{\circ}$ & $\begin{array}{c}\mathbf{Q} \\
{[\text { MVAr] }}\end{array}$ \\
\hline TR2 & 13.2 & 210 & 1 & 4.8 \\
\hline TR3 & 13.2 & 210 & 2 & 9.6 \\
\hline
\end{tabular}

Moreover, these capacitors have reactors in serie to limit inrush currents of their own. In Table5 these characterístics are detailed.

TABLE5- Reactors data

\begin{tabular}{|c|c|c|c|c|}
\hline TR & $\begin{array}{c}\mathbf{U}_{\mathbf{n}} \\
{[\mathbf{k V}]}\end{array}$ & $\begin{array}{c}\mathbf{I}_{\mathbf{n}} \\
{[\mathrm{A}]}\end{array}$ & $\begin{array}{c}\mathbf{R} \\
{[\Omega]}\end{array}$ & $\begin{array}{c}\mathbf{L} \\
{[\mathrm{mH}]}\end{array}$ \\
\hline TR2 & 15 & 210 & 0.0018 & 0.018 \\
\hline TR3 & 15 & 210 & 0.0018 & 0.018 \\
\hline
\end{tabular}

\section{III.-ANALYSIS}

First we analyzed the frequency response in the following network points:

1. Equivalent connection system point in $132 \mathrm{kV}$, $Z_{s}(W)$.

2. Connection of different loads at substations in $13.2 \mathrm{kV}, Z_{1}(w), Z_{2}(w)$ y $Z_{3}(w)$. (Figure5) 
Then, harmonic sources were applied in the connection load points, above mentioned. These sources were modelled with current sources with a characteristic spectrum of some nonlinear load, defined by harmonic order, amplitude and phase.

The distotions obtained at network points of interest will be analyzed and the differences between the results with both load models will be established.

\section{IV.-SIMULATIONS}

\section{IV.1)Simulations with FS (Frequency Scan)}

Figure 5 shows the points where the frequency response of the network with both load models were obtained.

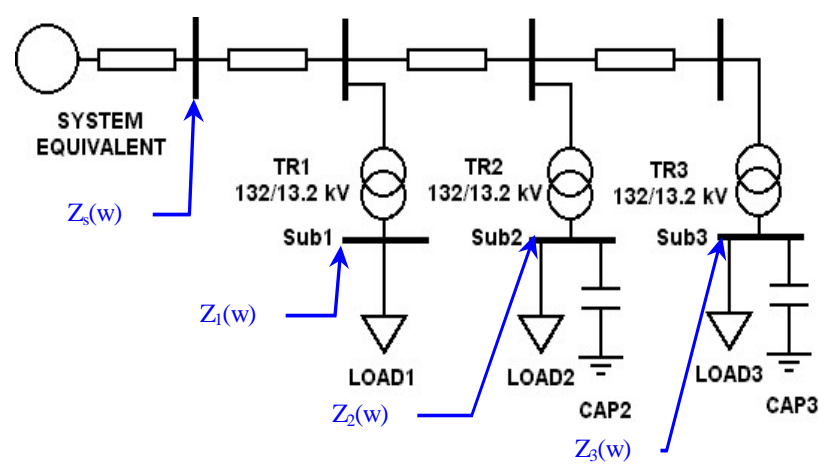

FIGURE5- Simulation de Z(w)

In Figure 6 it is shown the graph of $Z_{s}(w)$. In Figures 7, 8 and 9, it is shown the graphs de $Z_{1}(w)$, $Z_{2}(w)$ and $Z_{3}(w)$ also with both load models, respectively.

It can be seen the differences between both models like in the amplification of the values and in the resonance frequency in these Figures.

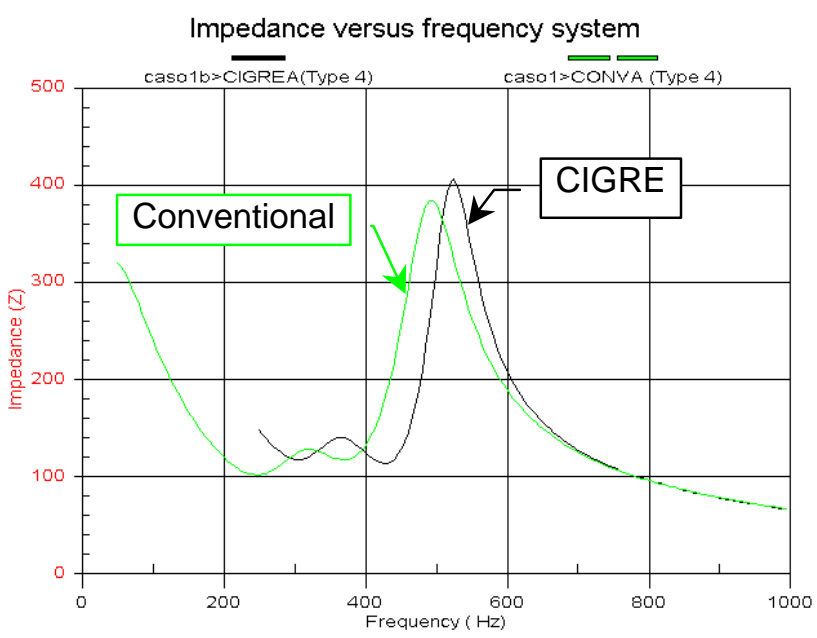

FIGURE6- $Z_{s}(w)$ with both models

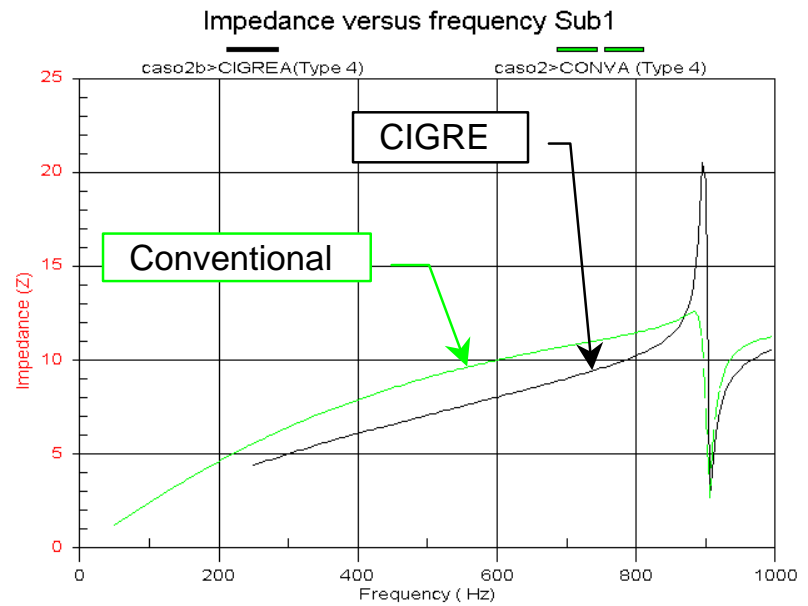

FIGURE7- $Z_{1}(w)$ with both models

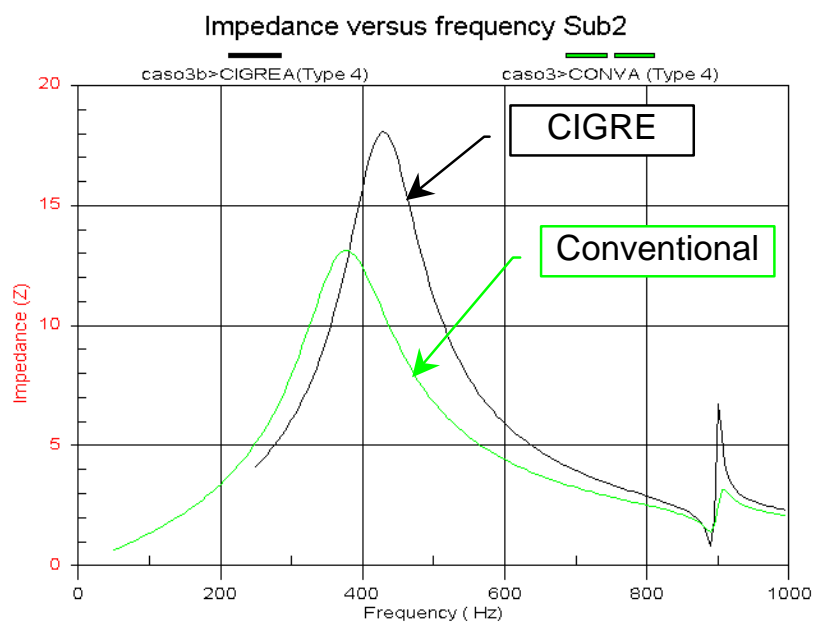

FIGURE8- $Z_{2}(w)$ with both models

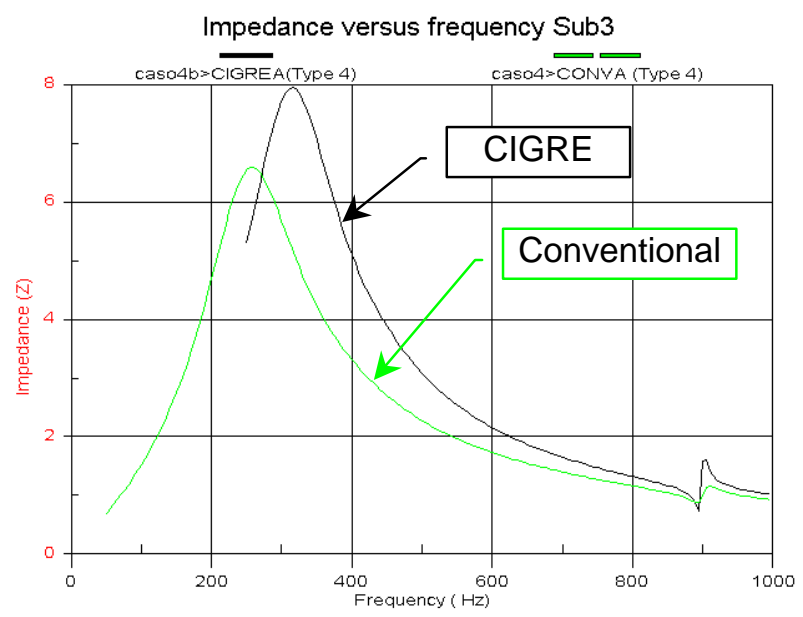

FIGURE9- $Z_{3}(w)$ with both models

The expression -[1]- was used to calculate the resonance frequency for CIGRE model in a network point, associated with the following values:

$$
f_{r}=f \sqrt{\frac{S_{c}}{Q_{c}}}
$$


where

$f_{r}$ : resonance frequency.

$f$ : nominal o main frequency system.

$S_{c}$ : shortcircuit power at system point.

$Q_{c}$ : reactive power of capacitor banks at that point.

In the substation 1, it is shown an amplification for high frequencies as in the substation there are no capacitor banks. The resonance is produced with the rest of the system. On the other hand, in the substation 2 it is produced a resonance around $400 \mathrm{~Hz}$. That can be verified replacing in -[1]- a value of $S_{c}$ established for the impedance of tranformer (see table2) and the value of $Q_{c}$ for substation 2 (see table4), resulting in:

$$
f_{r} \cong 50 H z \sqrt{\frac{320 M V A}{4.8 M v a r}}=408 H z
$$

Carrying out the same analysis for the substation 3 , it is verified:

$$
f_{r} \cong 50 H z \sqrt{\frac{300 M V A}{9.6 M v a r}}=280 H z
$$

Once the different frequency responses at interest system points are obtained, we get to know the troubles associated with the appearance of some harmonics. This can result from the own system $(132 \mathrm{kV})$ or from the loads in some substations $(13.2 \mathrm{kV})$.

Then harmonic currents will be only applied at user's connection points and the differences in the distortions obtained with both models will be analysed.

\section{IV.2) Simulations with HFS (Harmonic Frequency Scan)}

To define the spectrum and amplitudes of currents it was taken into account the installation of six-pulse controlled rectifier for each substation, with an active power of $10 \mathrm{MW}$. Through -[2]- the value of nominal current of rectifier can be calculated in order to establish the amplitudes of harmonic currents:

$$
\begin{aligned}
& P_{\text {act }}=\sqrt{3} \cdot U_{1} I_{1} \cos \varphi \\
& I_{1}=\frac{P_{\text {act }}}{\sqrt{3} \cdot U_{1} \cdot \cos \varphi}
\end{aligned}
$$

where

$P_{\text {act: }}$ value of active power.

$U_{i}$ : line voltage system.

$I_{1}$ : nominal line current

$\operatorname{Cos} \varphi$ : power factor of the load.
The rectifier which establishes a current spectrum like it is shown in Table 6, was considered ideal, supplied by a balance voltage system and without considering the phase, (see (2)),.

TABLE6- Values of currents

\begin{tabular}{|c|c|c|}
\hline \multicolumn{3}{|c|}{ Harmonic Source } \\
\hline Ih & Amp. & Amp.[A] \\
\hline $\mathrm{I}_{1}$ & $\mathrm{I}_{1}$ & 514.6 \\
\hline $\mathrm{I}_{5}$ & $\mathrm{I}_{1} / 5$ & 102.9 \\
\hline $\mathrm{I}_{7}$ & $\mathrm{I}_{1} / 7$ & 73.5 \\
\hline $\mathrm{I}_{11}$ & $\mathrm{I}_{1} / 11$ & 46.8 \\
\hline $\mathrm{I}_{13}$ & $\mathrm{I}_{1} / 13$ & 39.6 \\
\hline $\mathrm{I}_{17}$ & $\mathrm{I}_{1} / 17$ & 30.3 \\
\hline $\mathrm{I}_{19}$ & $\mathrm{I}_{1} / 19$ & 27.1 \\
\hline
\end{tabular}

The values of voltage obtained for each frequency will be the result from the multiplication provided in the ecuation -[3]-, that is to say to multiply the amplitudes of currents for the value of impedance obtained in IV.1) for the same harmonic.

$$
\left[V_{h}\right]=\left[I_{h}\right] x\left[Z_{h}\right] h=1, \ldots 20
$$

In Figures 10, 11 and 12 the results obtained from simulations are shown.

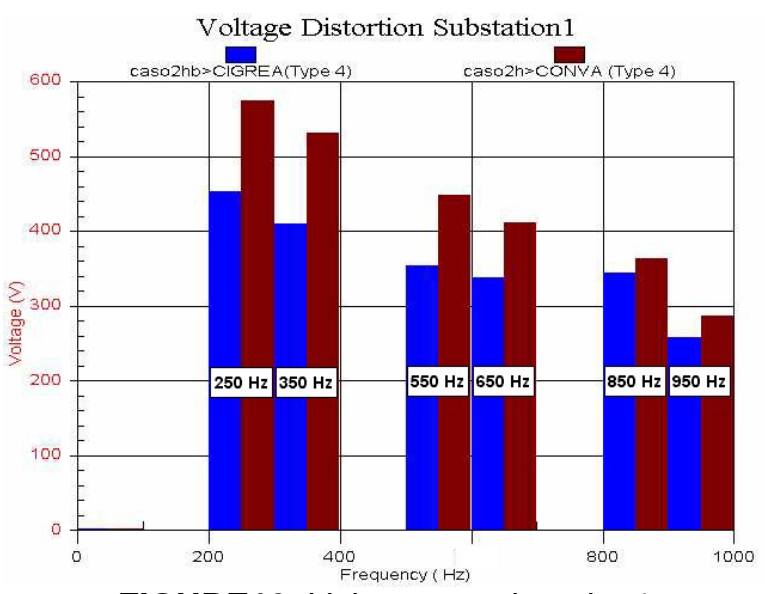

FIGURE10- Voltage at substation1

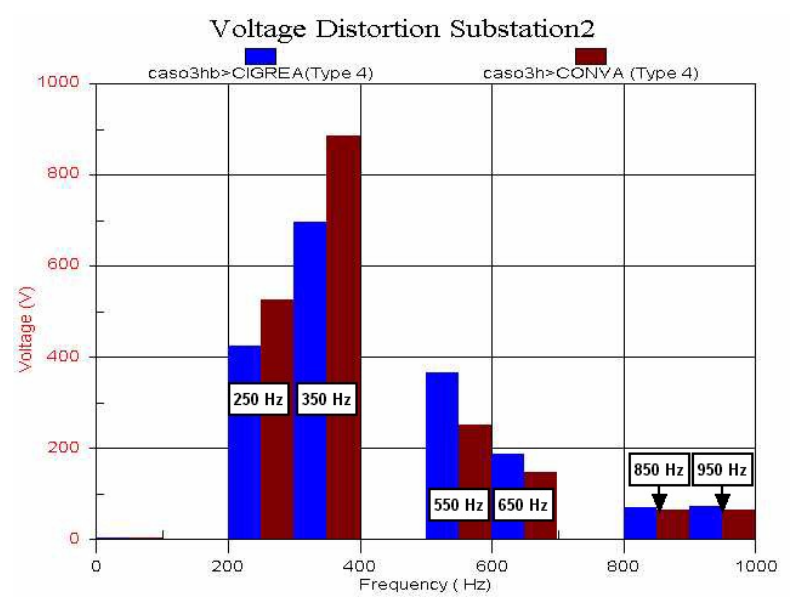

FIGURE11- Voltage at substation2 
The graphs show the distortions found for the voltages in all substations. The blue bars correspond to CIGRE load model and the brown bars correspond to conventional model.

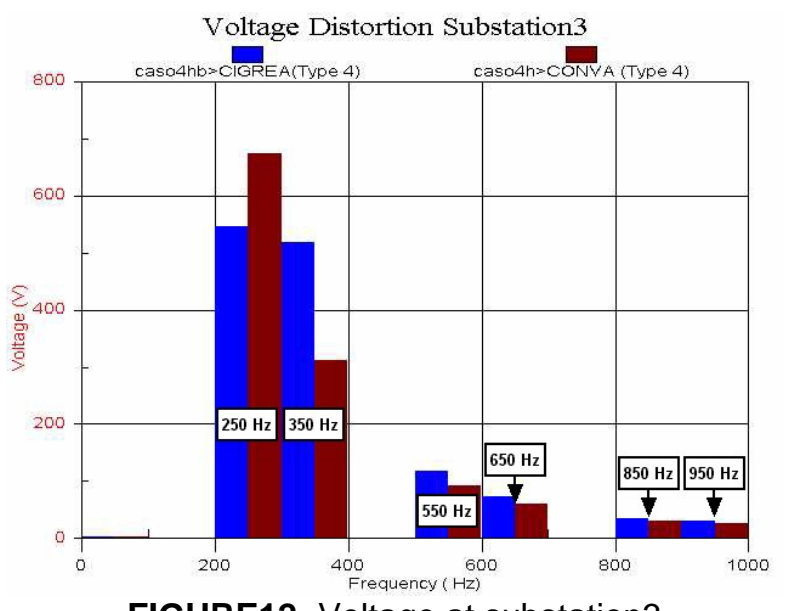

FIGURE12- Voltage at substation3

To establish the differences in the results obtained from both models, the factors established in -[4]- $y$ -[5]- which determine the distortion degree for voltage waveform are calculated.

$$
H F_{u}(\%)=\frac{U_{h}}{U_{1}} \times 100
$$

where

$H F_{u}:$ Harmonic Factor voltage of order $h$.

$U_{h}$ : harmonic amplitude voltage factor of order $h$.

$U_{1}$ : nominal harmonic amplitude of voltage.

$$
T H D_{u}(\%)=\frac{\sqrt{\sum_{u=2}^{\infty} U_{h}^{2}}}{U_{1}} \times 100
$$

where

$$
\text { THD : Total Harmonic Distortion voltage. }
$$

Supposing the amplitude voltage to nominal frequency is $13.2 \mathrm{kV}$, the values of Tables 7,8 and 9 are obtained.

It can be observed the diferences in the distortions obtained with both models in the results. The differences in THD are higher in two first cases due to the high differences obtained in $Z_{1}(w)$ y $Z_{2}(w)$

\begin{tabular}{|c|c|c|c|c|}
\hline \multicolumn{5}{|c|}{ Substation 1} \\
\hline \multirow{2}{*}{$\mathrm{h}$} & CIGRE & Conventional & CIGRE & Conventional \\
\hline & Voltage (V) & Voltage (V) & HF(\%) & HF(\%) \\
\hline 5 & 452 & 574 & 3,42 & 4,35 \\
\hline 7 & 410 & 530 & 3,11 & 4,02 \\
\hline 11 & 353 & 448 & 2,67 & 3,39 \\
\hline 13 & 337 & 412 & 2,55 & 3,12 \\
\hline 17 & 343 & 363 & 2,60 & 2,75 \\
\hline 19 & 270 & 280 & 2,05 & 2,12 \\
\hline & & THD & 6,78 & 8,27 \\
\hline
\end{tabular}
with respect to $Z_{3}(W)$, (see IV.1).

TABLE7- Distortioning comparison

\begin{tabular}{|c|c|c|c|c|}
\hline \multicolumn{5}{|c|}{ Substation 2} \\
\hline \multirow{2}{*}{$\mathrm{h}$} & CIGRE & Conventional & CIGRE & Conventional \\
\hline & Voltage $(\mathrm{V})$ & Voltage (V) & HF(\%) & HF(\%) \\
\hline 5 & 424 & 526 & 3,21 & 3,98 \\
\hline 7 & 697 & 886 & 5,28 & 6,71 \\
\hline 11 & 365 & 251 & 2,77 & 1,90 \\
\hline 13 & 188 & 148 & 1,42 & 1,12 \\
\hline 17 & 71 & 64 & 0,54 & 0,48 \\
\hline 19 & 70 & 65 & 0,53 & 0,49 \\
\hline & & THD & 6,96 & 8,14 \\
\hline
\end{tabular}

TABLE8- Distortioning comparison

\begin{tabular}{|c|c|c|c|c|}
\hline \multicolumn{5}{|c|}{ Substation 3} \\
\hline \multirow{2}{*}{$\mathrm{h}$} & CIGRE & Conventional & CIGRE & Conventional \\
\hline & Voltage (V) & Voltage (V) & HF(\%) & HF(\%) \\
\hline 5 & 546 & 673 & 4,14 & 5,10 \\
\hline 7 & 518 & 311 & 3,92 & 2,36 \\
\hline 11 & 118 & 92 & 0,89 & 0,70 \\
\hline 13 & 74 & 61 & 0,56 & 0,46 \\
\hline 17 & 35 & 32 & 0,27 & 0,24 \\
\hline 19 & 30 & 25 & 0,23 & 0,19 \\
\hline & & THD & 5,81 & 5,69 \\
\hline
\end{tabular}

TABLE9- Distortioning comparison

\section{V.- CONCLUSIONS}

When studies or simulation of harmonics are carried out, it is important to use some load model which has parameter variation with frequency, such as the suggested CIGRE model, because there are evident differences between the distortions.

In the Regulations of our country, (see (3)), limits are established for each Harmonic Factor (HF) from $0.2 \%$ to $6 \%$ and also limits for Total Harmonic Distortion (THD) which is $8 \%$ for medium level voltage. Therefore the differences of $1.5 \%$ y $1.18 \%$ found between both models, (see Table 7 and 8 respectively), can result from overcoming those levels in the network.

\section{V.- REFERENCIAS}

1. CIGRE Working Group 36-05 (Disturbing Loads),1981, "Harmonics, characterisitic parameters, methods of study, estimates of existing values in the network".

Electra, 77, 35-54.

2. J. Arrillaga, D. A. Bradley y P. S. Bodger (1985), Power System Harmonics.

3. Regulations ENRE 465/96, Metodologic Base for control of Quality Técnic Product. 